Letters to the Editor

NEUROSURGICAL FORUM

\section{Deep venous thrombosis and pulmonary embolism}

TO THE EDITOR: It is with great interest that we have read the clinical article by Rolston et al..$^{10}$ (Rolston JD, Han SJ, Bloch O, et al: What clinical factors predict the incidence of deep venous thrombosis and pulmonary embolism in neurosurgical patients? J Neurosurg 121:908-918, October 2014). They have investigated risk factors for venous thromboembolisms (VTEs) in neurosurgical patients using a large data set acquired from the American College of Surgeons' National Surgical Quality Improvement Program database over a 5-year period. ${ }^{10}$ In $1.7 \%$ of the 38,058 neurosurgical cases they observed, VTE became clinically evident within 30 days after surgery. Due to the apparent risks of intracranial hemorrhages, neurosurgeons are overly cautious in their use of perioperative anticoagulant and/ or antithrombotic agents. Nevertheless, the complications of a VTE, such as deep venous thrombosis or pulmonary embolism, have great impact on outcome in terms of morbidity and mortality. In a recent study we observed 90-day overall mortality rates of $5.0 \%$ and $23.1 \%$ when deep venous thrombosis and pulmonary embolism, respectively, occurred in patients who had undergone cranial meningioma resection. ${ }^{8}$

Interestingly, the authors established steroid use as a risk factor for VTE. They suggested this might be due to "a variety of increased procoagulant factors." 10 Indeed, there are some reports corroborating these findings and suggesting that steroids produce a hypercoagulable state in patients. ${ }^{4,11,12}$ Since the landmark report of Galicich et al. in 1961, high-dose corticosteroids are routinely administered to patients undergoing cranial surgery. ${ }^{7,9}$ More recently, a renewed interest in the use of corticosteroids for both the medical treatment and perioperative medical treatment of chronic subdural hematoma (CSDH) has been sparked., ${ }^{2,5,6}$ This usage raises some major questions when considering the potential VTE complications. Several comments on the use of glucocorticoids and the risk of VTE should be made, despite the findings of Rolston et al.

First, Rolston et al. could not define, and therefore not investigate, steroid use, because the pathology they investigated is subject to significant variation. Moreover, there is no information on dose and duration of treatment.
Second, to date, there is only one extensive review of literature by van Zaane et al. that attempts to shed light on this topic. ${ }^{13}$ However, the findings therein are tempered, as the authors state, by the lack of high-quality randomized trials. Nevertheless, they have shown that glucocorticoids tend both to lower the levels of several procoagulant factors, such as von Willebrand factor and fibrinogen, and simultaneously to give rise to an impaired fibrinolytic activity through increasing the levels of plasminogen activator inhibitor-1 (PAI-1). Thus, the net result of these mechanistic actions of corticosteroids on the coagulation system is not necessarily prothrombotic, or at least not adding to the thrombotic risk imposed by the surgery itself. ${ }^{13}$ Third, in a previous study, ${ }^{3}$ we observed that in patients who underwent surgery for $\mathrm{CSDH}$, and who were concomitantly treated with high-dose glucocorticoids, the incidence of VTE was $1.8 \%$, which supports the findings of Rolston et al. ${ }^{10}$ In these patients, we have found that both VTE and mortality were in fact not related to the duration of high-dose corticosteroid use. On the contrary, in patients operated on for intracranial meningiomas, all treated with perioperative corticosteroids, the occurrence of VTE was 7.2\%. ${ }^{8}$ This difference in VTE occurrence between these two conditions, with the same corticosteroid regimen, probably reflects the patient's hypercoagulable state in tumor surgery and thus underlines the importance of the pathology at hand rather than corticosteroid administration itself. ${ }^{1,8}$

Considering the above, we believe that further research is needed to assess the risk-benefit ratio of glucocorticoid use in neurosurgical practice, which requires a well-designed randomized controlled trial and clinical outcome studies. Additionally, we strongly suggest that VTE complications should be assessed as a major outcome, related to mortality and quality of life, in such studies to assess the effects of glucocorticoid treatment in a dose- and timedependent manner.

Victor Volovici, MD

Ruben Dammers, MD, PhD

Lotte M. E. Berghauser Pont, MD, MSc

Erasmus MC, Rotterdam, The Netherlands

DISCLOSURE

The authors report no conflict of interest. 


\section{References}

1. Anderson JA, Weitz JI: Hypercoagulable states. Crit Care Clin 27:933-952, 2011

2. Berghauser Pont LM, Dirven CM, Dippel DW, Verweij BH, Dammers R: The role of corticosteroids in the management of chronic subdural hematoma: a systematic review. Eur J Neurol 19:1397-1403, 2012

3. Berghauser Pont LME, Dammers R, Schouten JW, Lingsma H, Dirven CMF: Clinical factors associated with outcome in chronic subdural hematoma: a retrospective cohort study of patient on preoperative corticosteriod therapy. Neurosurgery 70:873-880, 2012

4. Calvo-Alén J, Toloza SM, Fernández M, Bastian HM, Fessler BJ, Roseman JM, et al: Systemic lupus erythematosus in a multiethnic US cohort (LUMINA). XXV. Smoking, older age, disease activity, lupus anticoagulant, and glucocorticoid dose as risk factors for the occurrence of venous thrombosis in lupus patients. Arthritis Rheum 52:2060-2068, 2005

5. Coulter IC, Kolias AG, Marcus HJ, Ahmed AI, Alli S, AlMahfoudh R, et al: Proposal for a prospective multi-centre audit of chronic subdural haematoma management in the United Kingdom and Ireland. Br J Neurosurg 28:199-203, 2013

6. Emich S, Richling B, McCoy MR, Al-Schameri RA, Ling $\mathrm{F}$, Sun L, et al: The efficacy of dexamethasone on reduction in the reoperation rate of chronic subdural hematoma-the DRESH study: straightforward study protocol for a randomized controlled trial. Trials 15:6, 2014

7. Galicich JH, French LA, Melby JC: Use of dexamethasone in treatment of cerebral edema associated with brain tumors. J Lancet 81:46-53, 1961

8. Hoefnagel D, Kwee LE, van Putten EH, Kros JM, Dirven CM, Dammers R: The incidence of postoperative thromboembolic complications following surgical resection of intracranial meningioma. A retrospective study of a large single center patient cohort. Clin Neurol Neurosurg 123:150-154, 2014

9. McClelland S III, Long DM: Genesis of the use of corticosteroids in the treatment and prevention of brain edema. Neurosurgery 62:965-968, 2008

10. Rolston JD, Han SJ, Bloch O, Parsa AT: What clinical factors predict the incidence of deep venous thrombosis and pulmonary embolism in neurosurgical patients? J Neurosurg 121:908-918, 2014

11. Stolz E, Klötzsch C, Schlachetzki F, Rahimi A: High-dose corticosteroid treatment is associated with an increased risk of developing cerebral venous thrombosis. Eur Neurol 49:247-248, 2003

12. van der Pas R, de Bruin C, Leebeek FW, de Maat MP, Rijken DC, Pereira AM, et al: The hypercoagulable state in Cushing's disease is associated with increased levels of procoagulant factors and impaired fibrinolysis, but is not reversible after short-term biochemical remission induced by medical therapy. J Clin Endocrinol Metab 97:1303-1310, 2012

13. van Zaane B, Nur E, Squizzato A, Gerdes VE, Büller HR, Dekkers OM, et al: Systematic review on the effect of glucocorticoid use on procoagulant, anti-coagulant and fibrinolytic factors. J Thromb Haemost 8:2483-2493, 2010

\section{Response}

No response was received from the authors of the original article.

\section{INCLUDE WHEN CITING}

Published online January 9, 2015; DOI: 10.3171/2014.9.JNS142030.

(CAANS, 2015

\section{Readmission}

TO THE EDITOR: We read with great interest recent articles on readmissions by Buchanan et al. ${ }^{1}$ [Buchanan CC, Hernandez EA, Anderson JM, et al: Analysis of 30day readmissions among neurosurgical patients: surgical complication avoidance as key to quality improvement. J Neurosurg 121:170-175, July 2014] and Marcus et al. ${ }^{4}$ [Marcus LP, McCutcheon BA, Noorbakhsh A, et al: Incidence and predictors of 30-day readmission for patients discharged home after craniotomy for malignant supratentorial tumors in California (1995-2010). J Neurosurg 120:1201-1211, May 2014], both published within the last several months in the Journal. In addition, Shah et al. ${ }^{5}$ [Shah MN, Stoev IT, Sanford DE, et al: Are readmission rates on a neurosurgical service indicators of quality of care? J Neurosurg 119:1043-1049, October 2013] had published a similar study in 2013 . The readmission rate is a commonly used quality measure, even though whether it actually leads to quality improvement has been challenged. ${ }^{3}$ The rates can appear to be significantly different depending on the study methodologies, and one way to differentiate them is from the standpoint of big data.

The rise of big data has been a topic of many excellent essays. ${ }^{2}$ Some of the characteristics of the studies at the two extremes of the data size spectrum are shown in Fig. 1. While the surgical literature is traditionally filled with case series near the right end of the spectrum, more and more policy-making decisions and studies are based on large databases. However, there is a trade-off between having pristine data and data drawn from a large sample size. This is particularly troubling for the clinicians, who, more than anyone else, are aware that clinical and social nuances are what make each patient unique and often influence outcome. Some of these factors have not yet been quantified (i.e., surgeon fatigue), and some are quantified with rudimentary scales (i.e., ASA physical status classification system). Finally, the utility of these databases hinges on information being entered accurately, which is not always the case.

Along this line of thinking, here are a few specific comments on the aforementioned studies:

1. In the study by Marcus et al., ${ }^{4}$ which utilized a statewide database to examine readmissions after supratentorial tumor resection $(n=18,506)$, they found that seizure was a main cause of readmission based on readmission ICD-9 codes. Do the authors have enough confidence in the validity of seizure diagnoses in this kind of database to recommend targeted prospective intervention?

2. Shah et al. ${ }^{5}$ and Buchanan et al. ${ }^{1}$ gathered detailed information on patients who were readmitted $(n=407$ and $n$ $=365$, respectively) in their respective institutions. Information was not gathered to the same extent for the rest of the patients in the cohort who were not readmitted $(n=3552$ and $n=5569)$. The detailed descriptions enabled the authors to offer their expert opinions on adjudicating preventability, an important topic of its own. However, in the absence of direct comparison between patients with and without the adverse events (readmis- 


\author{
Applicable to many patients \\ Heterogeneous population \\ Statistics: $\mathrm{n}=$ all \\ Looking for correlations
}

\section{Applicable to less patients Homogeneous population \\ Statistics: $\mathrm{n}=$ sampling \\ Looking for causation}

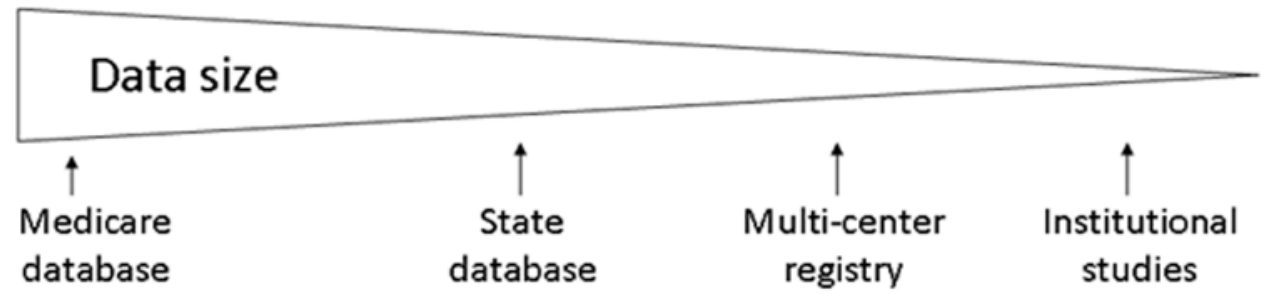

FIG. 1. Diagram showing characteristics of the studies at the two extremes of the data size spectrum.

sion), potentially meaningful and useful correlations may not come to light. For example, Shah et al. mentioned that insurance type did not seem to influence the likelihood of readmission $(p=0.09)$, but how about race/ ethnicity, urgency of intervention (emergency vs elective surgery), and procedure start time?

3. Buchanan et al. ${ }^{1}$ analyzed both adult and pediatric patients together. They found that CSF shunt malfunction was the second most likely reason for index admission and that its related complications accounted for the largest portion of readmitted patients. It is likely that the complication occurrences inversely correlate with patient age, but one cannot be sure if the analysis does not include all the other CSF shunt-treated patients who did not experience complications. Stratifying patients by age is perhaps one of the easier ways to increase data purity at some expense to the data sample size.

Prospective multiinstitutional data registries such as ones by the Hydrocephalus Clinical Research Network (HCRN) and National Neurosurgery Quality and Outcomes Database $\left(\mathrm{N}^{2} \mathrm{QOD}\right)$ Lumbar Deformity Module may avoid some of the pitfalls at both ends of the datasize spectrum. These databases offer distinct advantages, including a prospective cohort design, sufficient data size, a focus on data fidelity, and data collection geared toward predetermined clinical questions. However, the main concerns here are that these are treatment-based registries; therefore, patient selection was not taken into account, and the cost is high (T. Asher, presentation at the Annual Meeting of the American Association of Neurological Surgeons, 2014 [http://www.neuropoint.org/NPA\%20 N2QOD\%20Video.html]).

Despite some public misconception, physicians care about delivering efficient care no less than the hospital administration, the government, or the insurance industry. All of the authors should be commended for their efforts in further improving care even though the excellence of their individual institutions is already well known. Big data is a new instrument that has been made available in the Internet age, and its proper usage will become apparent only through repeated and judicious use over time.

Samir Sarda, BS ${ }^{1,2}$ Wei Dong, MS, MPH',2 Joshua J. Chern, MD, PhD ${ }^{1,2}$
${ }^{1}$ Pediatric Neurosurgery Associates, Children's Healthcare of Atlanta, Atlanta, GA

²Emory University, Atlanta, GA

DISCLOSURE

The authors report no conflict of interest.

\section{References}

1. Buchanan CC, Hernandez EA, Anderson JM, Dye JA, Leung M, Buxey F, et al: Analysis of 30-day readmissions among neurosurgical patients: surgical complication avoidance as key to quality improvement. J Neurosurg 121:170-175, 2014

2. Cukier K, Mayer-Schoenberger V: The rise of big data: how it's changing the way we think about the world. Foreign Aff 92:28-40, 2013

3. Joynt KE, Jha AK: Thirty-day readmissions-truth and consequences. N Engl J Med 366:1366-1369, 2012

4. Marcus LP, McCutcheon BA, Noorbakhsh A, Parina RP, Gonda DD, Chen C, et al: Incidence and predictors of 30-day readmission for patients discharged home after craniotomy for malignant supratentorial tumors in California (19952010). J Neurosurg 120:1201-1211, 2014

5. Shah MN, Stoev IT, Sanford DE, Gao F, Santiago P, Jaques DP, et al: Are readmission rates on a neurosurgical service indicators of quality of care? J Neurosurg 119:1043-1049, 2013

\section{Response}

We want to thank Sarda and colleagues for a balanced commentary on the strengths and weaknesses of big data as it relates to clinical research. The authors eloquently discussed the trade-off between having a pristine, validated data set (e.g., a clinical trial or highly annotated registry) and a data set with a large sample size. We feel these two types of data can be highly complementary in advancing clinical care.

One of big data's greatest strengths for clinical studies is in its ability to represent clinical associations (between diseases and patient, hospital, and provider factors) in a very generalizable way, avoiding the necessary selection bias that is involved in creating homogeneous populations for comparison that is required in smaller, highly curated data sets such as clinical trials. On the flip side, the most common criticism about clinical big data studies is their data inaccuracy. Administrative coding involves a large amount of data, entered by many different people with 
different training and experience, at different hospitals, at different points in time, and there is bound to be some data inconsistency and inaccuracy. However, these data inaccuracies, while fatal for smaller data sets, are not as fatal for big data as one may think.

Data inaccuracy comes in many forms. Some types of coding inaccuracies (e.g., data entry errors) are likely to be randomly distributed across the population and, as such, would be unlikely to affect studies that have a properly defined hypothesis, with proper comparison groups being analyzed. Other forms of data inaccuracy could reflect an inability to be granular in coding and not permit the accurate capture of important disease variation. This will likely to be improved in the transition from ICD-9 to ICD10. However, even if specific granular disease codes are available for use, a coder could still fail to record a true diagnosis (lowering the negative predictive value [NPV] by increasing the number of false negatives) or could inaccurately code a patient as having the condition of interest (lowering the positive predictive value [PPV] by increasing the number of false positives). Both of these errors increase the background "noise" when evaluating relationships of interest.

In the case of coding for seizures in administrative databases, there is some evidence that this latter form of data inaccuracy is relatively small. Jetté et al. ${ }^{1}$ showed that the PPV and NPV for ICD-9-CM coding of seizures from an emergency room database were, respectively, $99 \%$ and $97 \%$ and from a discharge abstract database were $98 \%$ and $99 \%$. The PPV and NPV for ICD-10 coding from the emergency room database were, respectively, $100 \%$ and $90 \%$ and from the discharge abstract database were $98 \%$ and $99 \%$.

Thus, in our study, an engineering metaphor may apply; if a positive signal (seizures) is detected in the midst of background noise (an admixture of properly and improperly coded patients), we can be certain that it is true and that the true signal would be even easier to detect if there were less noise (via more accurate coding). Data inaccuracy in the type of analysis that we have presented essentially works as a form of conservative bias: While we may not be able to detect all the positive associations that are out there, for the ones we do detect, we can be fairly certain about them.

In the case of our particular finding of a high number of seizures associated with readmission, we feel that a case can be made to more specifically evaluate, in smaller, better curated data sets, other relevant questions. Examples of these questions are as follows: Can a subset of patients at particular high risk be identified? Is the current frequency of administration of postsurgery seizure prophylaxis in this population sufficient to prevent those seizures that prompt readmission? Would further education and followup monitoring programs to ensure compliance with prescribed antiseizure medication programs decrease the rate of readmission? With these questions we come full circle, where "big data" helps frame the proper questions for the detailed "small data" studies of specific interventions.

David C. Chang, PhD, MPH, MBA
Logan P. Marcus, MS
Clark C. Chen, MD, PhD
Bob S. Carter, MD, PhD
University of California, San Diego, CA

\section{Reference}

1. Jetté N, Reid AY, Quan H, Hill MD, Wiebe S: How accurate is ICD coding for epilepsy? Epilepsia 51:62-69, 2010

\section{Response} et al.

No response was received from Buchanan et al. or Shah

INCLUDE WHEN CITING

Published online January 16, 2015; DOI: 10.3171/2014.6.JNS141417.

CAANS, 2015 